\title{
INVERSE MODEL FOR THE SOLUTE MICRO-FIELD FORMATION DURING SELF-PROPAGATING HIGH TEMPERATURE REACTION
}

\begin{abstract}
A new thermodynamic description for the self-propagating high temperature synthesis (SHS - reaction) is presented in the "inverse" version. This description is worked out for the diffusion barrier, thickness of which is at the limit, i.e. its value is infinitesimally small. The solution to the diffusion equation delivered in the description can be easily extended for the diffusion barrier of a greater thickness. The Ni/Al multi-layers system is treated as a virtual eutectic alloy solidifying with the rate equal to that involved by the self-propagating reaction. It is suggested to inverse the curves obtained for solidification in order to characterize the melting completed by the formation of the $\mathrm{AlNi}$ - intermetallic phase required in the self-propagating synthesis.

Keywords: diffusion equation; diffusion barrier; self-propagating synthesis
\end{abstract}

\section{Introduction}

Usually, the Ni/Al physical vapor deposited (PVD method) multilayers are subjected to self - propagating high temperature synthesis (SHS), [1]. It is assumed that the reaction occurs under steady-state and at a constant temperature. However, it is expected that the intermixing of nickel and aluminum at the multilayer interfaces, taking place during deposition, results in nucleation of two intermetallic phases i.e.: $\mathrm{Al}_{3} \mathrm{Ni}_{2}$, and $\mathrm{Al}_{3} \mathrm{Ni}$. The appearance of the mentioned phases is justified thermodynamically and additionally by the Phase Rule applied for the isothermal / isobaric formation of this complex, [2]. These intermetallic phases form a kind of a diffusion barrier and complicate the SHS - reaction. It was also proved that the intermetallic phases formation is the result of both $\mathrm{Ni}-$ substrate dissolution and subsequent meta-stable solidification of the dissolved zone which became liquid, [3]. In fact, the metastable dissolution of aluminum in the $\mathrm{Ni}$ - substrate leads to the formation of the liquid film in the $\mathrm{Ni}$ - sub-layers, as justified thermodynamically, Fig. 1. Although, the solidification begins in stable condition, it transforms immediately into meta-stable solidification according to the thermodynamic criterion formulated for the appearance / disappearance of the peritectic reaction during investigated processes, [4].

According to the mentioned criterion, and Phase Rule, the existence of the $\mathrm{AlNi}$ - phase in the diffusion barrier is not possible. Instead of that, the meta-stable $\mathrm{Al}_{3} \mathrm{Ni}_{2}$ - phase is created, [3]. The formation of the $\mathrm{Al}_{3} \mathrm{Ni}_{2}$ - intermetallic phase as the result of peritectic reaction between $\mathrm{AlNi}$ and adequate liquid would be possible during the initial transient stable solidification, only.
Consequentially, the meta-stable solidification of the $\mathrm{Al}_{3} \mathrm{Ni}_{2}-$ phase occurs according to the phenomenon of partitioning. On the other hand, the formation of the $\mathrm{Al}_{3} \mathrm{Ni}$ - compound is the result of the undercooled peritectic reaction during both initial transient stable and final meta-stable solidifications.

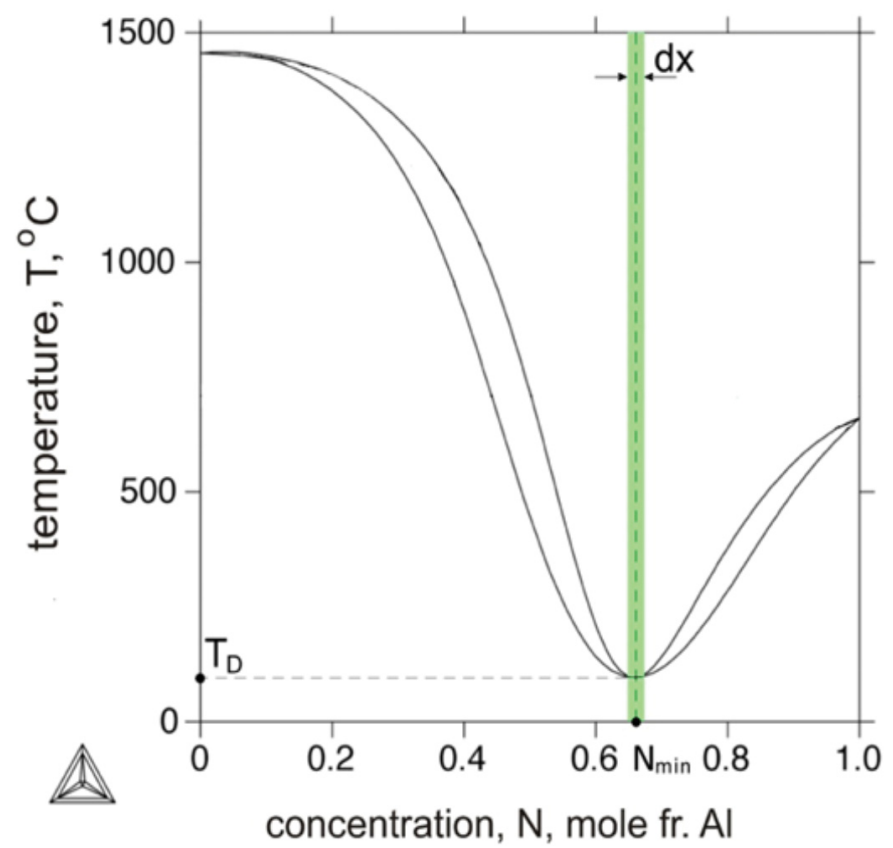

Fig. 1. Ni-Al phase diagram for meta-stable dissolution of aluminum in nickel; formation of the $d x$ - liquid film in the nickel is possible at the $\mathrm{T}_{\mathrm{D}}\left(\sim 80^{\circ} \mathrm{C}\right)$; the diagram justifies that the dissolution should occur at the liquidus minimum, for $\mathrm{N}_{\min }=66$ at.\% $\mathrm{Al}$ (Thermocalc Software calculation)

\footnotetext{
* INSTITUTE OF METALLURGY AND MATERIALS SCIENCE, POLISH ACADEMY OF SCIENCES, REYMONTA 25, 30 059 KRAKÓW, POLAND

\# Corresponding author: w.wolczynski@imim.pl
} 
The aim of the current model is to develop an "inverse", thermodynamic model for the self-propagating reaction (synthesis) when the diffusion barrier is at the limit, that is, its thickness is at the limit, i.e. its value is infinitesimally small. The development of the "inverse" model is possible / justified since melting and solidification are opposed phenomena. Instead of studying melting, the "inverse" model gives a more effective tool to apply the diffusion equation. The use of the diffusion equation is adequate to show how two hypothetical eutectic "ingredients" appear during solidification.

\section{Solution to the diffusion equation}

The diffusion equation for the "inverse" model of the selfpropagation reaction is as follows:

$$
\frac{d C(x, z)}{d z}=-\frac{D\left(T_{\max }\right)}{v} \nabla^{2} C(x, z)
$$

$T_{\max }-$ maximum temperature reached during steady-state propagation of the reaction,

$v$ - steady-state rate of the self-propagation reaction (determined theoretically, [1]),

$D$ - diffusion coefficient in the liquid,

$C-$ Ni solute concentration in the liquid ahead of the s/l interface.

$$
v^{2}=\frac{3 A \exp \left(-E /\left(R T_{\max }\right)\right)}{\left(\delta^{\prime}\right)^{2} E\left(T_{\max }-T_{0}\right)} R T_{\max }{ }^{2} \lambda^{2}
$$

A - the Arrhenius factor,

$E$ - activation energy for the mass diffusion,

$R$ - gas constant,

$\delta^{\prime}-1 / 4$ of the bilayer thickness (the sum of the $A$ and $B$ layer thicknesses $\delta$,

$T_{0}$ - initial temperature of the system.

As the self-propagating reaction occurs (in first approximation) at constant rate, it involves the following simplification: $D\left(T_{\max }\right) \approx$ const. This assumption is justified since, practically, the $T_{\max }$ - temperature does not vary during the process under investigation, [1].

The diffusion equation is formulated for the $\mathrm{Ni}-\mathrm{Al}$ - system considered as the virtual eutectic system, Fig. 2, with the eutectic point corresponding to the $\mathrm{Ni}$ - solute concentration in the $\mathrm{AlNi}$ - phase (created by the self-propagating reaction), Fig. 3a.

The hypothetical eutectic phase diagram, Fig. 2, is simplified (sophisticated) but useful in the current consideration. However, in the more realistic situation the AlNi - phase should exhibit its melting temperature higher than the melting point for the $\mathrm{N}^{\mathrm{F}}$ - solution. The $\mathrm{N}^{\mathrm{F}}$ - equilibrium solution is always formed due to the nickel diffusion into liquid aluminum, [3]. It is not possible to preserve pure aluminum in such a system, [3].

The current solution to the diffusion equation, Eq. (1), is given for the "inverse" problem, that is, for the situation when

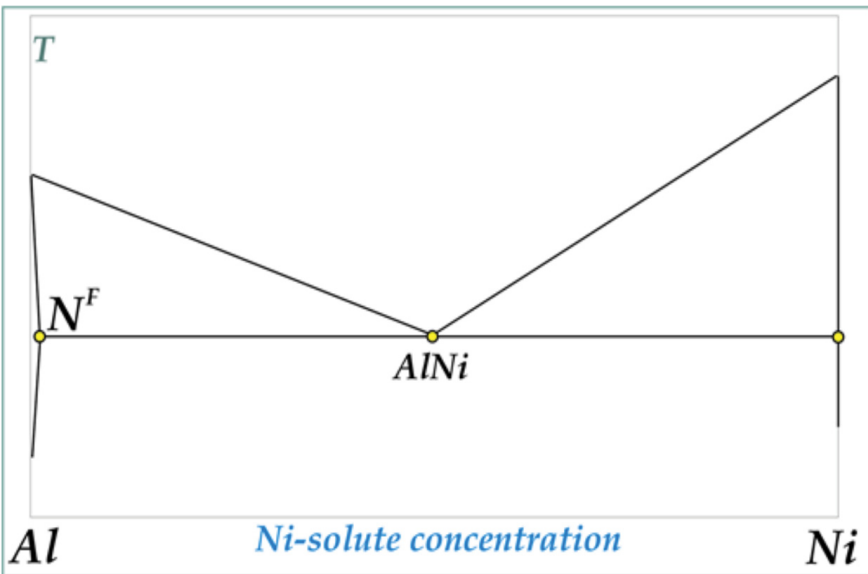

Fig. 2. Hypothetical eutectic phase diagram used for the solution of the diffusion equation, Eq. (1)

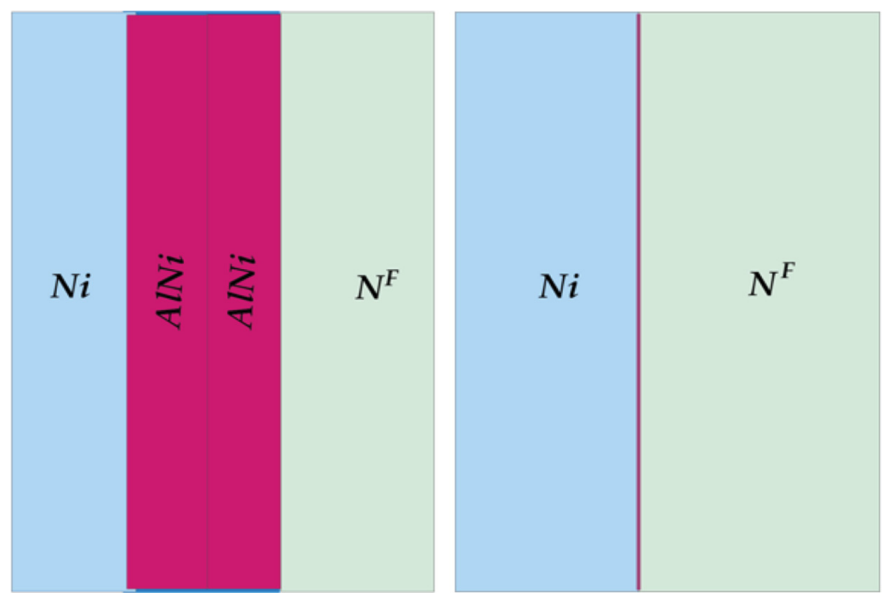

Fig. 3. The $\mathrm{Ni}$ - sub-layer /diffusion barrier/ $\mathrm{N}^{\mathrm{F}}$ - sub-layer system; a) with the diffusion barrier transformation into the required / final phase; b) without the diffusion barrier transformation (at the diffusional limit), just before the self-propagating reaction; $\mathrm{N}^{\mathrm{F}}$ - equilibrium solution of nickel in aluminum, [2]

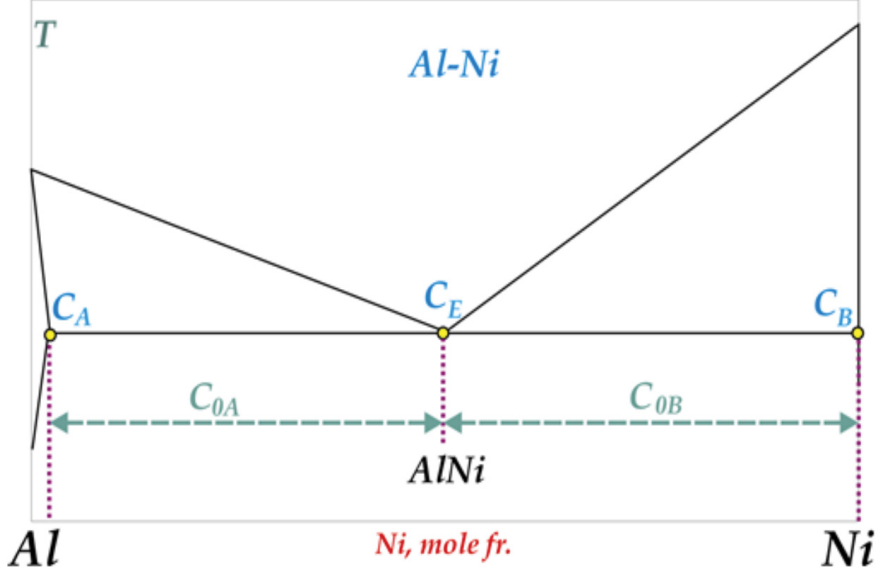

Fig. 4. Definitions of some parameters used in the current solution

the liquid AlNi- phase disperses into two lamellae, i.e.: $\mathrm{Ni}$, and $\mathrm{N}^{\mathrm{F}}$, hypothetically, Fig. 3b, Fig. 4. However, it is worth to remind that the self-propagating reaction (synthesis) is as follows: $\mathrm{Ni}+\mathrm{N}^{\mathrm{F}} \rightarrow$ AlNi. 
The Lever Rule is fulfilled as follows: $\frac{C_{0 A}}{C_{0 B}}=\frac{\lambda_{N i}}{\lambda_{N F}}$, Fig. 4.

$\lambda_{N F}$ - width of the $\mathrm{Al}\left(\mathrm{N}^{\mathrm{F}}\right)$ - lamella,

$\lambda_{N i}$ - width of the $\mathrm{Ni}$ - lamella,

$T_{\max }=$ const., since, $v=$ const.,

Usually, $T_{\max }=1273^{\circ} \mathrm{C}$, [5]. Therefore, when, $v=$ const.,

$$
\frac{d C(x, y, z)}{d t}=-D \nabla^{2} C(x, y, z)
$$

Additionally, for the steady-state solidification, when $d z / d t=v$ is valid, and for the lamellar growth, Fig. 5:

$$
\begin{aligned}
& \frac{d C(x, y, z)}{d t} \frac{d z}{d z}=-D \nabla^{2} C(x, y, z) \\
& \frac{d C(x, z)}{d z}=-\frac{D\left(T_{\max }\right)}{v} \nabla^{2} C(x, z)
\end{aligned}
$$

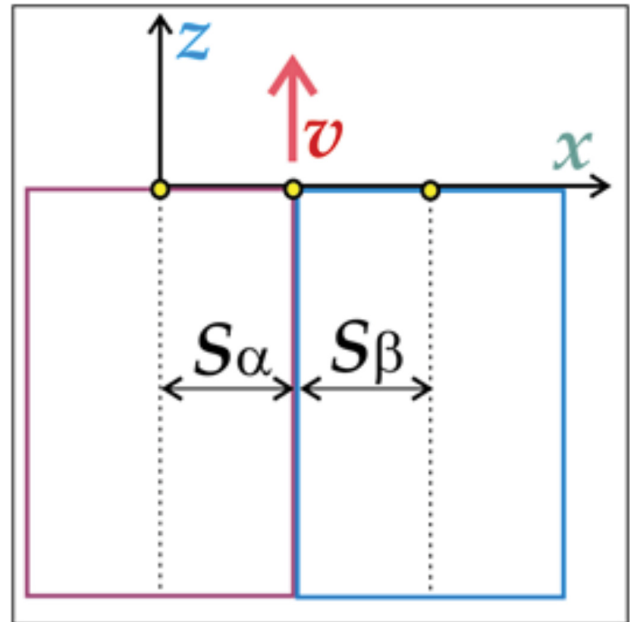

Fig. 5. Co-ordinate system attached to $\mathrm{s} / \mathrm{l}$ interface of the solidifying eutectic

$S_{\alpha}$ - half the width of the $\mathrm{N}^{\mathrm{F}}-$ lamella; $S_{\beta}-$ half the width of the $\mathrm{Ni}$ - lamella. Thus,

$$
\frac{C_{0 A}}{C_{0 B}}=\frac{S_{\beta}}{S_{\alpha}}
$$

Finally, the diffusion equation is:

$$
\frac{d(\delta C(x, z))}{d z}+\frac{D}{v} \nabla^{2}(\delta C(x, z))=0
$$

Consequentially, the $\Delta T_{N i}$, and $\Delta T_{N F}$ - undercooling appear. Moreover,

$$
\Delta T_{N i} \neq \Delta T_{N F}
$$

The first, well known solutions to diffusion equation, [6]-[8], do not take into account Eq. (7). However, this very important and fruitful in consequences assumption given by Eq. (7) has already been inserted into a quite recent solution to the diffusion equation, [9].
In the current model the diffusion equation is given in two dimensions only:

$$
\frac{\partial^{2} \delta C}{\partial x^{2}}+\frac{\partial^{2} \delta C}{\partial z^{2}}+\frac{v}{D} \frac{\partial \delta C}{\partial z}=0
$$

The solution of this equation can be shown as a product of two functions, i.e.:

$$
\delta C(x, z)=X(x) Z(z)
$$

with

$$
\begin{gathered}
Z(z)=\exp \left[\left(-\frac{v}{2 D}-\sqrt{\frac{v^{2}}{4 D^{2}}+\omega^{2}}\right) z\right] \\
X(x)=A \cos (\omega x)+B \sin (\omega x)
\end{gathered}
$$

Some boundary conditions are as follows, Fig. 4, Fig. 5:

$$
\begin{gathered}
\frac{\partial C}{\partial x}=0, \text { for } x=0, \text { and for } x=S_{\alpha}+S_{\beta} \\
\delta C\left(S_{\alpha}, z\right)=C\left(S_{\alpha}, z\right)-C_{E}=0 \\
\frac{\partial C}{\partial z}=\mp \frac{v C_{0 J}}{D} ;(J=A, B) \text { for } z=0 \\
\text { and } 0 \leq x<S_{\alpha} ; S_{\alpha}<x \leq S_{\alpha}+S_{\beta}
\end{gathered}
$$

After some modifications and for the plane $\mathrm{s} / \mathrm{l}$ interface, Fig. 5:

A/ for the $\mathrm{N}^{\mathrm{F}}$ (generally, for the $\alpha$ - eutectic phase), that is for $x \in\left[0, S_{\alpha}\right], z \geq 0$, the value of the $B$, and $\omega$-parameters results from the following condition:

$$
\left.\frac{\partial \delta C(x, z)}{\partial x}\right|_{x=0}=0
$$

It is obvious, Eq. (15), that: $-\omega A \sin (\omega \cdot 0)+\omega B \cos (\omega \cdot 0)$ $=0$, which yields $B=0$. It also yields, Eq. (15), Eq. (13), that: $A \cos \left(\omega S_{\alpha}\right)=0 \omega=\omega_{2 n-1}=\frac{(2 n-1) \pi}{2 S_{\alpha}}, n=1,2, \ldots$.

Combining Eq. (9), Eq. (10), and Eq. (11) it can be written (with the use of Eq. (13) and Eq. (15)) that solution to Eq. (8), is:

$$
\begin{aligned}
\delta C(x, z)= & \sum_{n=1}^{\infty} A_{2 n-1} \cos \left(\frac{(2 n-1) \pi x}{2 S_{\alpha}}\right) \\
& \exp \left[\left(-\frac{v}{2 D}-\sqrt{\frac{v^{2}}{4 D^{2}}+\left(\frac{(2 n-1) \pi}{2 S_{\alpha}}\right)^{2}}\right) z\right]
\end{aligned}
$$

where, $A_{2 n-1}$, are constant.

Assuming that the $v=$ const. is rather low velocity, one can assume that:

$$
\frac{(2 n-1) \pi}{2 S_{\alpha}}>>\frac{v}{2 D}
$$


144

hence, for the self - propagating reaction (synthesis):

$$
\delta C(x, z)=\sum_{n=1}^{\infty} A_{2 n-1} \cos \left(\frac{(2 n-1) \pi x}{2 S_{\alpha}}\right) \exp \left(-\frac{(2 n-1) \pi}{2 S_{\alpha}} z\right)
$$

The following conditions are to be applied in order to define the $A_{2 n-1}$ - parameter:

$$
\left.\frac{\partial \delta C(x, z)}{\partial z}\right|_{z=0}=f_{\alpha}(x) ; \quad f_{\alpha}(x)<0, \quad x \in\left[0, S_{\alpha}\right]
$$

then:

$$
\begin{aligned}
\left.\frac{\partial \delta C(x, z)}{\partial z}\right|_{z=0}= & \sum_{n=1}^{\infty} A_{2 n-1}\left(-\frac{v}{2 D}-\sqrt{\frac{v^{2}}{4 D^{2}}+\left(\frac{(2 n-1) \pi}{2 S_{\alpha}}\right)^{2}}\right) \\
& \cos \left(\frac{(2 n-1) \pi x}{2 S_{\alpha}}\right)
\end{aligned}
$$

and for the self-propagating reaction:

$$
\left.\frac{\partial \delta C(x, z)}{\partial z}\right|_{z=0}=\sum_{n=1}^{\infty} A_{2 n-1}\left(-\frac{(2 n-1) \pi}{2 S_{\alpha}}\right) \cos \left(\frac{(2 n-1) \pi x}{2 S_{\alpha}}\right)
$$

It is worth to note that the introduced function $f(x)$, has the following properties:

$$
\begin{aligned}
& f(x), \quad-2 S_{\alpha} \leq x \leq 2 S_{\alpha}, \\
& f(-x)=f(x), \quad f\left(x+2 S_{\alpha}\right)=-f(x) \\
& f(x) \approx \frac{a_{0}}{2}+\sum_{n=1}^{\infty} a_{n} \cos \left(\frac{n \pi x}{2 S_{\alpha}}\right) \\
& a_{n}=\frac{1}{S_{\alpha}} \int_{0}^{2 S_{\alpha}} f(x) \cos \left(\frac{n \pi x}{2 S_{\alpha}}\right) d x
\end{aligned}
$$

Assuming $f\left(x+2 S_{\alpha}\right)=-f(x)$, it yields: $a_{2 k}=0, k=0,1,2, \ldots$ for $n=2 k a_{2 k-1}=\frac{2}{S_{\alpha}} \int_{0}^{S_{\alpha}} f(x) \cos \left(\frac{(2 k-1) \pi x}{2 S_{\alpha}}\right) d x, k=1,2, \ldots$ for $n=2 k-1, k=1,2, \ldots$

Finally, the Fourier's series for $f(x)$ can be shown as:

$$
f(x) \approx \sum_{k=1}^{\infty} a_{2 k-1} \cos \left(\frac{(2 k-1) \pi x}{2 S_{\alpha}}\right)
$$

and after some rearrangements:

a/ for rapid solidification,

$$
\begin{aligned}
& A_{2 n-1}=\left(-\frac{v}{2 D}-\sqrt{\frac{v^{2}}{4 D^{2}}+\left(\frac{(2 n-1) \pi}{2 S_{\alpha}}\right)^{2}}\right)^{-1}, \\
& \frac{2}{S_{\alpha}} \int_{0}^{S_{\alpha}} f_{\alpha}(x) \cos \left(\frac{(2 n-1) \pi x}{2 S_{\alpha}}\right) d x \\
& n=1,2, \ldots
\end{aligned}
$$

b/ for self-propagating reaction,

$$
A_{2 n-1}=-\frac{4}{(2 n-1) \pi} \int_{0}^{S_{\alpha}} f_{\alpha}(x) \cos \left(\frac{(2 n-1) \pi x}{2 S_{\alpha}}\right) d x, n=1,2, \ldots
$$

The obtained solution, Eq. (16), Eq. (16*) as well as Eq. (23), Eq. $\left(23^{*}\right)$ satisfies:

$$
\left.\frac{\partial \delta C(x, z)}{\partial x}\right|_{x=0}=\left.\frac{\partial \delta C(x, z)}{\partial x}\right|_{x=2 S_{\alpha}}=0
$$

$$
\begin{gathered}
\left.\frac{\partial \delta C(x, z)}{\partial z}\right|_{z=0}=f_{\alpha}(x)=f_{\alpha}(-x)=-f_{\alpha}\left(-x+2 S_{\alpha}\right) \\
=-\left.\frac{\partial \delta C\left(-x+2 S_{\alpha}, z\right)}{\partial z}\right|_{z=0} \\
\\
\quad x \in\left[0, S_{\alpha}\right]
\end{gathered}
$$

according to the assumptions: $f_{\alpha}(-x)=f_{\alpha}(x), f_{\alpha}\left(x+2 S_{\alpha}\right)=-f_{\alpha}(x)$.

B/ for the Ni (generally, for the $\beta$ - eutectic phase), that is for: $x \in\left[S_{\alpha}, S_{\alpha}+S_{\beta}\right], z \geq 0$ :

a/ for rapid solidification,

$$
\begin{aligned}
\delta C(x, z) & =\sum_{n=1}^{\infty} B_{2 n-1} \cos \left(\frac{(2 n-1) \pi\left(x-S_{\alpha}+S_{\beta}\right)}{2 S_{\beta}}\right) \\
& \exp \left[\left(-\frac{v}{2 D}-\sqrt{\frac{v^{2}}{4 D^{2}}+\left(\frac{(2 n-1) \pi}{2 S_{\beta}}\right)^{2}}\right) z\right] \\
B_{2 n-1}= & \left.\left(-\frac{v}{2 D}-\sqrt{\frac{v^{2}}{4 D^{2}}+\left(\frac{(2 n+1) \pi}{2 S_{\beta}}\right)^{2}}\right)^{-1}\right] \\
& \frac{2}{S_{\beta}} \int_{S_{\alpha}-S_{\beta}}^{S_{\alpha}} f_{\beta}(x) \cos \left(\frac{(2 n+1) \pi\left(x-S_{\alpha}+S_{\beta}\right)}{2 S_{\beta}}\right) d x
\end{aligned}
$$

b/ for the self-propagating reaction,

$$
\begin{aligned}
\delta C(x, z)= & \sum_{n=1}^{\infty} B_{2 n-1} \cos \left(\frac{(2 n-1) \pi\left(x-S_{\alpha}+S_{\beta}\right)}{2 S_{\beta}}\right) \\
& \exp \left(-\frac{(2 n-1) \pi}{2 S_{\beta}} z\right)
\end{aligned}
$$

$$
\begin{aligned}
B_{2 n-1}= & -\frac{4}{(2 n-1) \pi} \\
& \int_{S_{\alpha}-S_{\beta}}^{S_{\alpha}} f_{\beta}(x) \cos \left(\frac{(2 n-1) \pi\left(x-S_{\alpha}+S_{\beta}\right)}{2 S_{\beta}}\right) d x \\
& n=1,2, \ldots \\
& \left.\frac{\partial \delta C(x, z)}{\partial z}\right|_{z=0}=f_{\beta}(x), \quad x \in\left[0, S_{\beta}\right]
\end{aligned}
$$




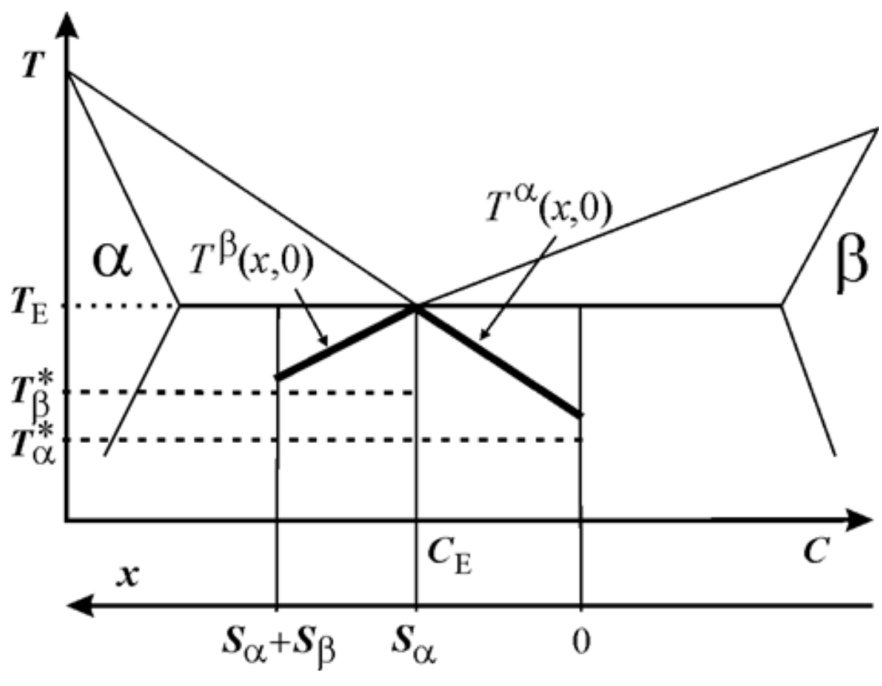

Fig. 6. Scheme of the eutectic phase diagram with the geometrical coordinate axis added in correlation with the co-ordinate system shown in Fig. 5 ( $x$-axis)

The obtained solution to the diffusion equation, Eq. (8), can be localized on the phase diagram under assumption that undercooling for the $\alpha$-phase is: $\Delta T_{\alpha}=T_{E}-T_{\alpha}{ }^{*}$, and for the $\beta$-phase is: $\Delta T_{\beta}=T_{E}-T_{\beta}^{*}$, Fig. 6 , where, $T_{E}$ - equilibrium eutectic temperature, $T_{\alpha}{ }^{*}, T_{\beta}{ }^{*}$-real temperature of the $\mathrm{s} / \mathrm{l}$ interface, respectively. This undercooling is described by the following functions: $T^{\alpha}(x, 0)$, and $T^{\beta}(x, 0)$, (bold lines $) ; T$ - temperature.

Consequentially, the current solution, Eq. $\left(16^{*}\right)$, and Eq. $\left(26^{*}\right)$ can also be shown in the $C(x, 0)$, and $T(x, 0)$ co-ordinate systems, Fig. 7.
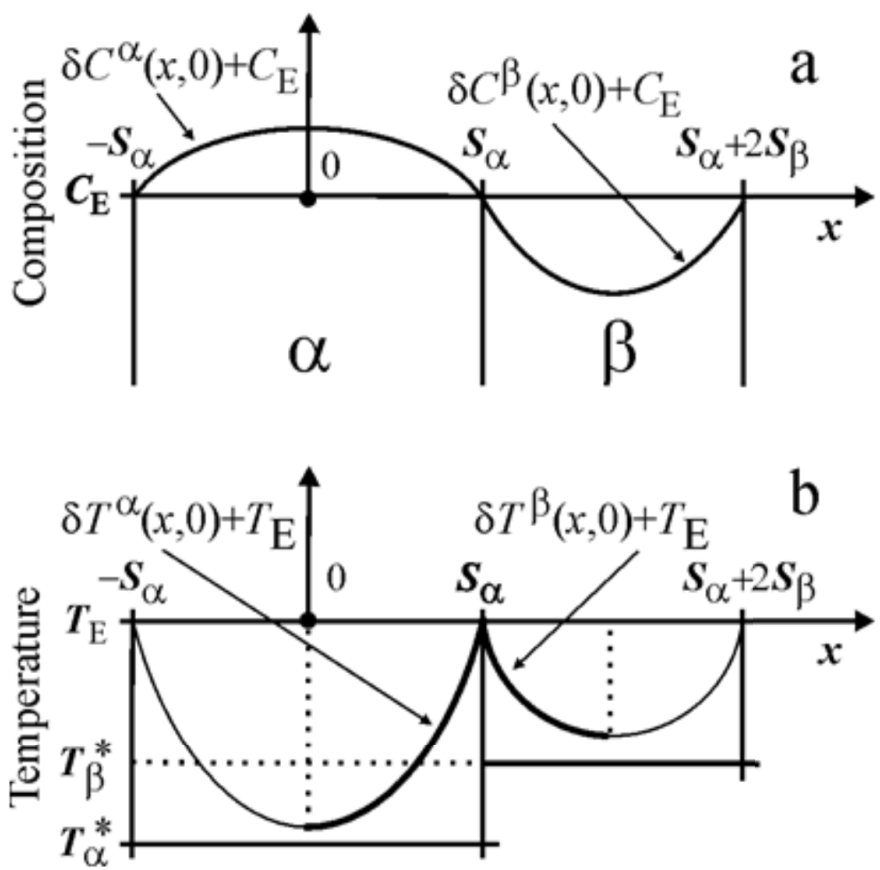

Fig. 7. Formal presentation of the current solution to the diffusion equation; for $z=0 ; \mathrm{a} / \mathrm{Ni}$ - solute concentration micro-field, $\mathrm{b} /$ real temperature micro-field, with the undercooling taken into account; both shown for a given solidification rate which is equal to $v=$ const. - rate of the self-propagating reaction

\section{Concluding remarks}

The obtained solution is to be verified by the mass balance in the $x, z$-system, i.e.:

$$
\begin{aligned}
& \int_{0}^{\infty} \int_{0}^{S_{\alpha}} \delta C(x, z) d x d z+\int_{0}^{\infty} \int_{S_{\alpha}}^{S_{\alpha}+S_{\beta}} \delta C(x, z) d x d z+= \\
& \sum_{n=1}^{\infty} \frac{4(-1)^{n-1} D}{(2 n-1) \pi}\left(\begin{array}{l}
\frac{A_{2 n-1} S_{\alpha}^{2}}{v S_{\alpha}+\sqrt{v^{2} S_{\alpha}^{2}+(2 n-1)^{2} D^{2} \pi^{2}}} \\
-\frac{B_{2 n-1} S_{\beta}^{2}}{v S_{\beta}+\sqrt{v^{2} S_{\beta}^{2}+(2 n-1)^{2} D^{2} \pi^{2}}}
\end{array}\right)=0
\end{aligned}
$$

Additionally, the correlation between $A_{2 n-1}$, and $B_{2 n-1}$ parameter can be defined:

a/ for rapid solidification,

$$
B_{2 n-1}=\frac{A_{2 n-1} S_{\alpha}^{2}\left(v S_{\beta}+\sqrt{v^{2} S_{\beta}^{2}+(2 n-1)^{2} D^{2} \pi^{2}}\right)}{S_{\beta}^{2}\left(v S_{\alpha}+\sqrt{v^{2} S_{\alpha}^{2}+(2 n-1)^{2} D^{2} \pi^{2}}\right)}, n=1,2, \ldots
$$

b/ for the self-propagating reaction,

$$
B_{2 n-1}=A_{2 n-1}\left(\frac{S_{\alpha}}{S_{\beta}}\right)^{2}, n=1,2, \ldots
$$

The global mass balance, Eq. (29) can be plotted as shown in Fig. 8.

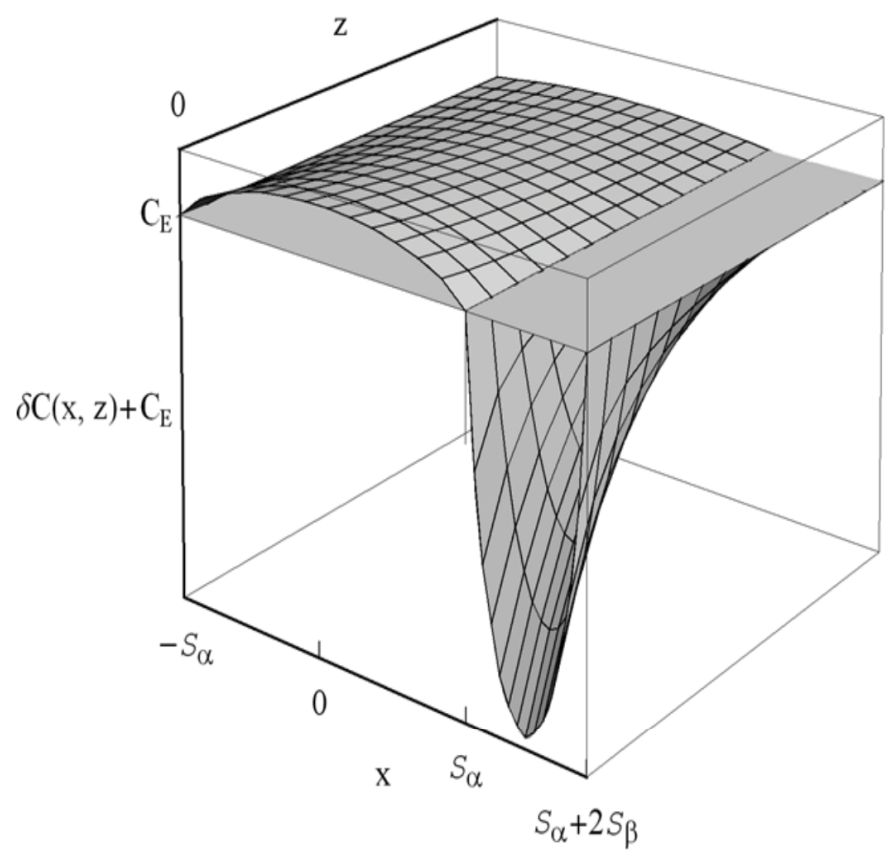

Fig. 8. Total mass balance for the considered eutectic system, Eq. (29)

Also, the local mass balance can be easily presented, Fig. 9 , according to Eq. (31). 


$$
\int_{0}^{S_{\alpha}} \delta C(x, 0) d x+\int_{S_{\alpha}}^{S_{\alpha}+S_{\beta}} \delta C(x, d) d x=0
$$

The local mass balance is satisfied under condition that the $d$-protrusion of the leading eutectic phase is taken into account, theoretically. It is justified since the leading phase protrusion was observed experimentally, [10], and comprehensively described from the thermodynamic viewpoint, [11]-[15].

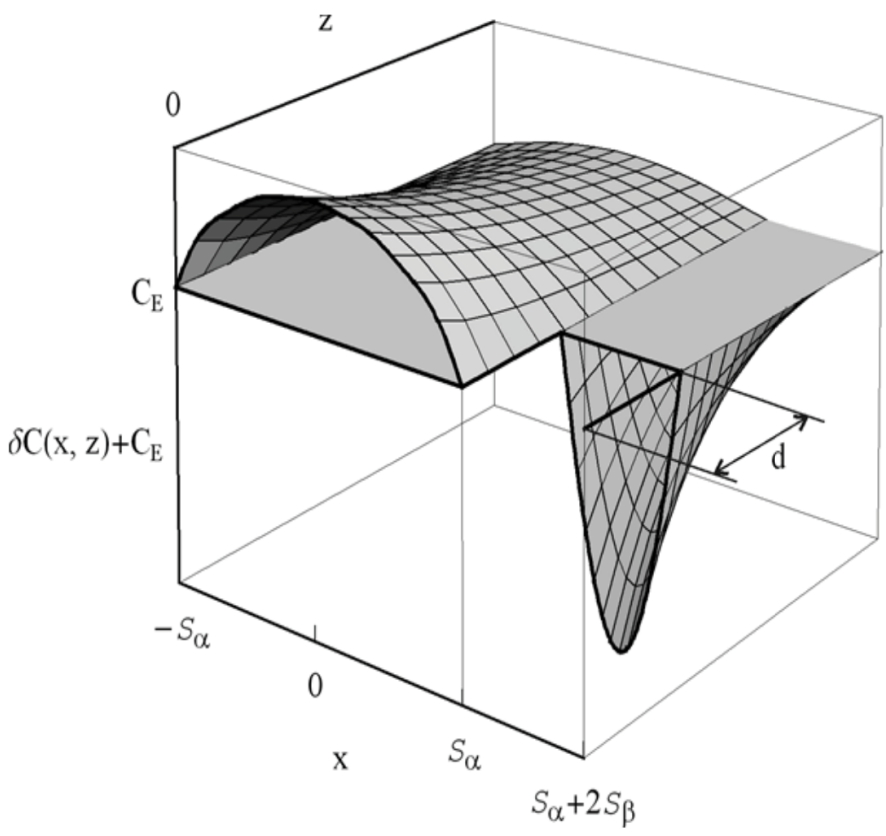

Fig. 9. Local mass balance for the studied eutectic system, Eq. (31)

Eq. (32) is also valid for the local mass balance:

$$
\begin{aligned}
& \sum_{n=1}^{\infty} A_{2 n-1} \frac{2 S_{\alpha}(-1)^{n-1}}{(2 n-1) \pi}-\sum_{n=1}^{\infty} B_{2 n-1} \frac{2 S_{\beta}(-1)^{n-1}}{(2 n-1) \pi} \\
& \exp \left(-\frac{v S_{\beta}+\sqrt{v^{2} S_{\beta}^{2}+(2 n-1)^{2} D^{2} \pi^{2}}}{2 D S_{\beta}} d\right)=0
\end{aligned}
$$

This relationship allows to deliver the theoretical definition for the $d$-phase protrusion which results from the current model, Eq. (31):

a/ for rapid solidification,

$$
\sum_{n=1}^{\infty} A_{2 n-1} \frac{(-1)^{n-1}}{(2 n-1)}\left(\begin{array}{c}
1-\frac{S_{\alpha}\left(v S_{\beta}+\sqrt{v^{2} S_{\beta}^{2}+(2 n-1)^{2} D^{2} \pi^{2}}\right)}{S_{\beta}\left(v S_{\alpha}+\sqrt{v^{2} S_{\alpha}^{2}+(2 n-1)^{2} D^{2} \pi^{2}}\right)} \\
\exp \left(-\frac{v S_{\beta}+\sqrt{v^{2} S_{\beta}^{2}+(2 n-1)^{2} D^{2} \pi^{2}}}{2 D S_{\beta}}\right)
\end{array}\right)=0
$$

b/ for the self-propagating reaction,

$$
\sum_{n=1}^{\infty} A_{2 n-1} \frac{(-1)^{n-1}}{(2 n-1)}\left(1-\frac{S_{\alpha}}{S_{\beta}} \exp \left(-\frac{(2 n-1) \pi}{2 S_{\beta}} d\right)\right)=0
$$

The theoretical justification for the existence of the $d$-protrusion at the SHS - reaction front (in the "inverse" model) has already been confirmed experimentally for the experiment of the self-propagating synthesis, [5], (the adequate scheme showing the protrusion of the leading phase has also been presented). The mentioned experimental disclosure of the protrusion formation, [5], confirms the cohesion between self-propagating reaction itself and the current "inverse" model for this technology.

The current "inverse" model describes solidification whereas the melting process occurs during self-propagating reaction. However, the current model has almost the same meaning as that which names melting as "inverse solidification", [17]. In fact, solidification is opposed phenomenon in comparison with melting. Therefore, it is sufficient to inverse the curves plotted in Fig. 7, Fig. 8, and Fig. 9, to characterize the melting phenomenon which occurs in the self-propagating reaction (synthesis).

The presented solution to the diffusion equation is developed for the infinitesimally small diffusion barrier. However, the current model can be easily expanded for the diffusion barrier of higher thickness. The future model for the diffusion barrier of higher thickness, following the present analysis, would be reducible perfectly, mathematically to the current model.

The current description is based on the thermodynamic predictions justifying that the diffusion barrier is formed in the meta-stable condition due to nickel sub-layer dissolution and subsequent solidification of the meta-stable phase, $\mathrm{Al}_{3} \mathrm{Ni}_{2}$, (according to partitioning between the $\mathrm{AlNi}$ - liquidus and meta-stable solidus of the $\mathrm{Al}_{3} \mathrm{Ni}_{2}$ - phase, Fig. 10). The $\mathrm{Al}_{3} \mathrm{Ni}$ - inter-metallic compound appears in sequence as a result of the peritectic reaction: $\mathrm{Al}_{3} \mathrm{Ni}_{2}+$ liquid $\rightarrow \mathrm{Al}_{3} \mathrm{Ni}$. The liquid film, essential for solidification, is formed due to meta-stable conditions set up for dissolution and subsequent directional growth of phases. The stable solidification which is created for a while, just before the dominant meta-stable solidification, is very important for the involved course of the directional growth of some phases, [18]. The formation of the liquid film / zone, Fig. 1, in this condition, is analogous / similar to a liquid film formation at the migrating interface created between primary and peritectic phases, [19].

The presented conclusions / predictions are in a good agreement with the observations by the transmission electron microscopy (TEM) dealing with the reaction controlled by the solid state diffusion at the Ni/Al multilayer interfaces, [20]. The reaction involves appearance of the same phases at the multilayer interfaces, as these predicted in the current analysis. It also shows intensive intermixing of aluminum and nickel during their deposition / settlement, [20]. 


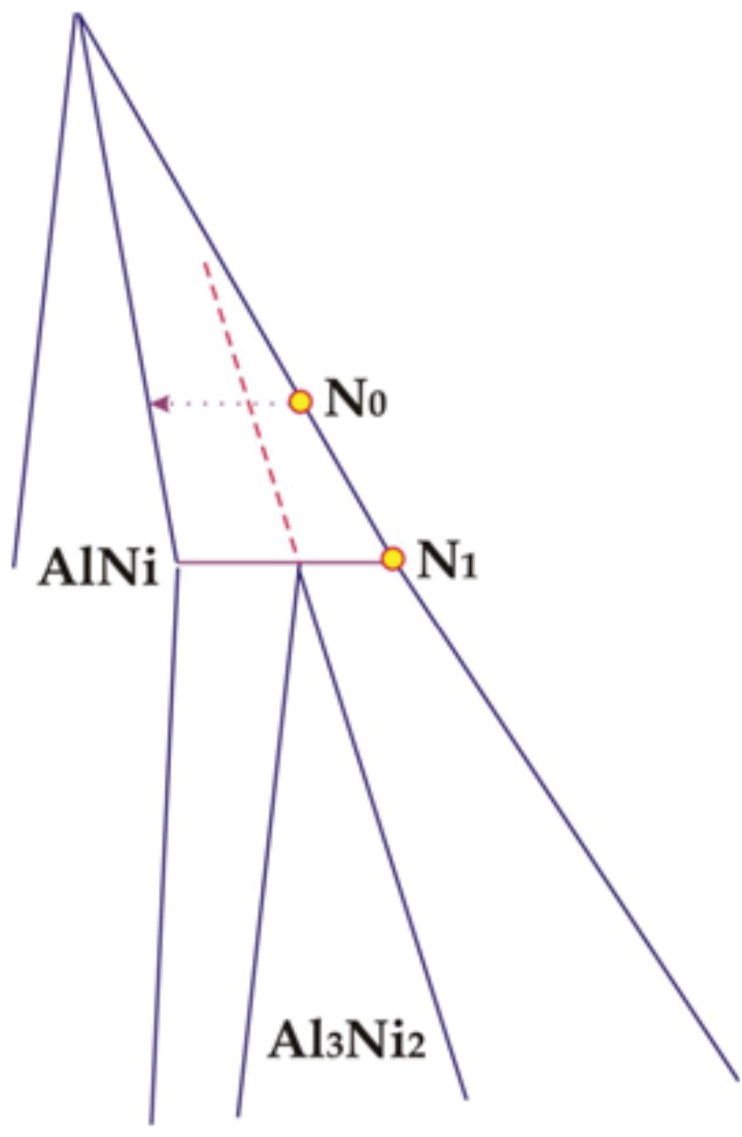

Fig. 10. Principle of the meta-stable formation of the $\mathrm{Al}_{3} \mathrm{Ni}_{2}$ - intermetallic phase instead of the expected stable AlNi - phase; $N_{0}$ - nominal solute concentration which is equal to the $N_{\min }$ - concentration, Fig. 1; instead of the partitioning between the $\mathrm{AlNi}$ - phase liquidus and solidus and subsequent peritectic reaction: $\mathrm{AlNi}+\mathrm{N}_{1} \rightarrow \mathrm{Al}_{3} \mathrm{Ni}_{2}$, the meta-stable partitioning is created between the $\mathrm{AlNi}$ - phase liquidus and the meta-stable solidus of the $\mathrm{Al}_{3} \mathrm{Ni}_{2}$ - phase (dashed line); thus the meta-stable $\mathrm{Al}_{3} \mathrm{Ni}_{2}$ phase forms instead of the stable $\mathrm{AlNi}$ - phase; in this case the process is more rapid and peritectic time-consuming reaction is excluded (the bottle-neck phenomenon); consequentially the solvus of the $\mathrm{Al}_{3} \mathrm{Ni}_{2}$ - phase is shifted towards the AlNi phase field.

\section{Acknowledgements}

The financial support was provided by the National Science Center (NCN), Poland under grant No. DEC-2012/05/B/ST8/01794.

\section{REFERENCES}

[1] A.J. Gavens, D. Van Heerden, A.B. Mann, M.E. Reiss, T.P. Weihs, J. Appl. Phys. 87, 1255-1263 (2000).

[2] W. Wołczyński, C. Senderowski, J. Morgiel, G. Garzeł, Arch. Metall. Mater. 59, 211-220 (2014).

[3] W. Wołczyński, Arch. Metall. Mater. 60 (3), 2421-2429 (2015).

[4] T. Umeda, T. Okane, W. Kurz, Acta Mater. 44, 4209-4216 (1996).

[5] A.S. Rogachev, Rus. Chem. Rev. 77, 21-37 (2008).

[6] K.A. Jackson, J.D. Hunt, Trans. Metall. Soc. AIME 236, 11291142 (1966).

[7] P. Magnin, R. Trivedi, Acta Metall. Mater. 39, 453-467 (1991).

[8] A.V. Catalina, S. Sen, D.M. Stefanescu, Metall. Mater. Trans. 34A, 383-394 (2003)

[9] W. Wołczyński, Def. Diff. Forum 272, 123-138 (2007).

[10] V.L. Davies, J. Inst. Met. 93, 10-14 (1964/5).

[11] W. Wołczyński, Cryst. Res. Technol. 25, 1303-1309 (1990).

[12] W. Wołczyński, Cryst. Res. Technol. 25, 1433-1437 (1990).

[13] W. Wołczyński, Cryst. Res. Technol. 26, 173-178 (1991).

[14] W. Wołczyński, Cryst. Res. Technol. 27, 121-125 (1992).

[15] W. Wołczyński, Cryst. Res. Technol. 27, 195-200 (1992).

[16] K.J. Blobaum, D. Van Heerden, A.J. Gavens, T.P. Weihs, Acta Mater. 51, 3871-3884 (2003).

[17] M. Rettenmayr, Int. Mater. Rev. 54, 1-17 (2009).

[18] H. Nguyen-Thi, B. Drevet, J.M. Debierre, B. Billia, J. Cryst. Growth 253, 539-548, (2003).

[19] P. Peng, L. Xinzhong, L. Jiangong, S. Yanqing, G. Jingjie, F. Hengzhi, Sci. Rep. 6, 24512, (2016).

[20] Ł. Maj, J. Morgiel, Thin Solid Films 621, 165-170 (2017). 